\title{
Inhibition of return interacts with the Simon effect: An omnibus analysis and its implications
}

\author{
JASON IVANOFF and RAYMOND M. KLEIN \\ Dalhousie University, Halifax, Nova Scotia, Canada \\ and \\ JUAN LUPIÁÑEZ \\ University of Granada, Granada, Spain
}

\begin{abstract}
Previous research has reported that the Simon effect (a type of stimulus-response [S-R] compatibility effect) and the inhibition of return effect (IOR; a late cuing effect) do not interact. In this brief report, we analyzed published and unpublished experiments that have examined these effects and found that IOR actually increases the Simon effect. This is a remarkable finding because most factors that delay reaction times (as IOR does) actually decrease the Simon effect. We examine this interaction within the context of seven interpretations of the effect that IOR may have on the task-irrelevant S-R code and two interpretations of the effect that IOR may have on the task-relevant S-R code, two components that underlie the Simon effect. The results falsified more than half of these interpretations, thus permitting future investigations to further reduce the number of theoretical alternatives.
\end{abstract}

Within certain task parameters, the sudden appearance of an irrelevant stimulus (a cue) in the periphery attracts attention (Posner, 1980). Reflecting this attraction, the processing of a subsequent, task-relevant stimulus (a target) is facilitated if it is near the cued location, so long as the time interval between the onsets of the successive stimuli is short. If the time interval between the cue and target is relatively long, reaction times (RTs) are usually slower for targets in the vicinity of the cue (Posner \& Cohen, 1984). This slowing of RT has been attributed to an inhibitory aftereffect that is initiated or becomes measurable when attention is removed from the cued location. Because it was thought that attention was inhibited from returning to the originally cued location, the inhibitory aftereffect has been called inhibition of return (IOR; see Klein, 2000, Lupiáñez, Tudela, \& Rueda, 1999, and Taylor \& Klein, 1998, for reviews). ${ }^{1}$ This interpretation was challenged by Klein and Taylor (1994), who suggested that the slowed RT might be due to a response bias, a reluctance to respond to targets at the cued location. Although evidence from a variety of sources supports a response contribution to the IOR effect (Abrams \& Dobkin, 1994; Fuentes, Vivas, \& Humphreys, 1999; Ivanoff \& Klein, 2001; Posner, Rafal, Choate, \& Vaughan, 1985; Taylor \& Klein, 2000), several investigators have rejected a response contribution on the basis of the failure of the IOR effect to interact significantly with the Simon effect (Lupiáñez, Milán, Tornay, Madrid,

Correspondence concerning this article should be addressed to J. Ivanoff, Department of Psychology, Dalhousie University, Halifax, NS, B3H 4J1 Canada (e-mail: ji @ or.psychology.dal.ca).
\& Tudela, 1997; Lupiáñez \& Milliken, 1999; Lupiáñez \& Solano, 1998; Pratt, Kingstone, \& Khoe, 1997). The purpose of this paper is to re-examine this issue. Before we do, however, we want to point out that different interpretations of the nature of IOR are not mutually exclusive. A result that rejects or supports a response contribution to the IOR effect would not, by itself, affect the view that IOR reflects attention or perceptual stages of processing.

The Simon effect refers to the performance advantage for responses that spatially correspond to the relative location of the target (target-response [T-R] correspondence) compared with responses that do not correspond ( $\mathrm{T}-\mathrm{R}$ noncorrespondence), when the spatial location of the target is task-irrelevant (see Lu \& Proctor, 1995, for a review). The general consensus is that the Simon effect occurs because of conflict or congruence between two spatial stimulus-response ( $\mathrm{S}-\mathrm{R})$ codes: one that is transiently generated from the spatial location of the target (and that is task-irrelevant) and one that, due to the task instructions, is activated by the nonspatial identity of the target (e.g., De Jong, Liang, \& Lauber, 1994; Hommel, 1993a, 1994; Kornblum, Stevens, Whipple, \& Requin, 1999). If the IOR effect is the result of response processes, then, following additive factors logic (Sternberg, 1969), it should interact with the Simon effect. In apparent conflict with this prediction, there have been repeated reports that Simon and IOR effects do not interact reliably (e.g., Lupiáñez et al., 1997; Lupiáñez \& Milliken, 1999; Lupiáñez \& Solano, 1998; Pratt et al., 1997).

The absence of a reliable interaction between the Simon effect and the IOR effect might be observed because the two effects are truly additive. According to additive factors logic, additivity entails the strong inference 
that the two effects operate at different stages of processing. Such an inference should be made with caution. Alternatively, the true relation might be an interaction whose nonsignificance could be due to a lack of statistical power. To disconfirm any proposal by accepting the null hypothesis is always risky given the possibility of committing a Type II error. Is there any reason to suspect a Type II error in the literature that has investigated the relationship between the IOR effect and the Simon effect? Lupiáñez et al. (1997) found that the IOR and Simon effects interacted significantly only with a $1,000-\mathrm{msec}$ cue-target onset asynchrony (CTOA). In this one experiment, the Simon effect was larger at the cued location than at the uncued location. Pratt et al. (1997) found a nonsignificant $(p=.09)$ interaction between the IOR and Simon effects, but the direction of the effect (i.e., larger Simon effect at the cued location) was consistent with that observed by Lupiáñez et al. (1997). Thus, it seems plausible that the IOR and Simon effects do interact, but that the null interaction results from low statistical power.

We conducted a power analysis in order to determine the sample size that would be required to find an interaction between IOR and Simon effects. Because the true effect size of the interaction (which will be represented mathematically and conceptually as the effect of IOR on the Simon effect $)^{2}$ is not known, we generated four hypothetical effect sizes. Figure 1 shows the degree of power (i.e., the proportion of experiments that would be expected to show a significant effect) as a function of sample size and effect size. Within the context of this paper, effect size is defined as the real absolute difference between the Simon effect for uncued and cued targets divided by the standard deviation of the difference (i.e., Cohen's $d$ ). Note that as the magnitude of the effect size increases, smaller sample sizes can detect significant effects. Further, smaller effects may only be detectable with large sample sizes. For example, with a sample size of 18 , only $13 \%$ of experiments would be expected to yield a statistically significant effect with an effect size of .2. At the other extreme, a very large difference in the Simon effect between cued and uncued RTs (Cohen's $d=.8$ ) could be detected by about $80 \%$ of experiments that used a sample size of only 15 . The general point to be made here is that if there is a true interaction between the Simon and IOR effects, but it is small in magnitude (yet theoretically meaningful), it will rarely be significant when relatively small samples are used.

\section{OMNIBUS ANALYSIS}

We examined the interaction between T-R compatibility (levels: correspondence and noncorrespondence) and cuing (levels: cued and uncued) at four different CTOAs $(100,400,700$, and $1,000 \mathrm{msec})$ across several experiments (Lupiáñez et al., 1997; Lupiáñez \& Milliken, 1999; Lupiáñez, Milliken, Solano, Weaver, \& Tipper, 2001; Lupiáñez \& Solano, 1998; Lupiáñez, Weaver, Tipper, \& Madrid, 2001; Pratt et al., 1997). Table 1 lists the experiments included in the analyses. Initially, each

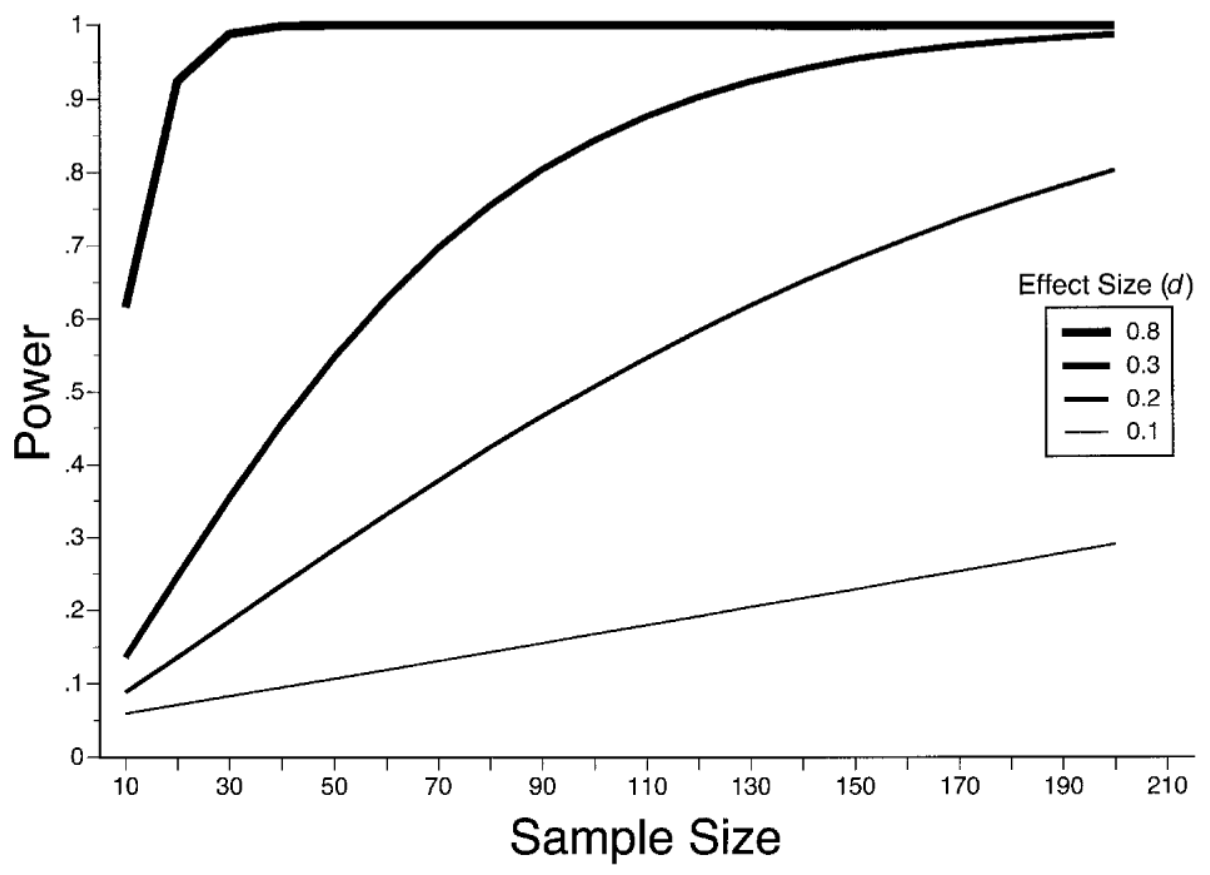

Figure 1. Power is shown as a function of sample size and effect size. Effect size (hypothetical effect sizes for the population) was calculated as the absolute value of the mean difference divided by the standard deviation of the difference. In this context, $.2, .5$, and .8 are small, medium, and large effect sizes, respectively. 
Table 1

A List of the Experiments Included in the Analyses

\begin{tabular}{|c|c|c|c|c|c|c|}
\hline Investigators & Experiment & Catch Trials & Type of Discrimination & $\begin{array}{l}\text { CTOAs } \\
\text { (in Milliseconds) }\end{array}$ & CTOA Manipulation & $N$ \\
\hline $\begin{array}{l}\text { Lupiáñez, Milan, } \\
\text { Tornay, Madrid, \& } \\
\text { Tudela (1997) }\end{array}$ & $1-5$ & $20 \%$ & Color & $\begin{array}{l}100,400 \\
700,1,000\end{array}$ & $\begin{array}{l}\text { All subjects did } 100 \mathrm{msec} \text {, } \\
\text { and one other CTOA } \\
\text { within a block of trials }\end{array}$ & 84 \\
\hline $\begin{array}{l}\text { Lupiáñez \& Solano } \\
\quad(1998)\end{array}$ & 1 & None & Color & $100,1,000$ & $\begin{array}{l}\text { All subjects did both } \\
\text { CTOAs }\end{array}$ & 16 \\
\hline $\begin{array}{l}\text { Lupiáñez \& Milliken } \\
\text { (1999) }\end{array}$ & $2 a, 2 b$ & $0 \%$ and $20 \%$ & Color & $\begin{array}{l}100,400 \\
700,1,000\end{array}$ & $\begin{array}{l}\text { All subjects did all } \\
\text { CTOAs within a block of } \\
\text { trials }\end{array}$ & 40 \\
\hline $\begin{array}{l}\text { Lupiáñez, Milliken, } \\
\text { Solano, Weaver, \& } \\
\text { Tipper (2001) }\end{array}$ & $1 b, 2$ & $0 \%$ and $20 \%$ & $\begin{array}{l}\text { Color and identity } \\
(\mathrm{X} / \mathrm{O})\end{array}$ & $\begin{array}{l}100,400 \\
700,1,000\end{array}$ & $\begin{array}{l}\text { All subjects did all } \\
\text { CTOAs between } \\
\text { different blocks of trials }\end{array}$ & 43 \\
\hline $\begin{array}{l}\text { Lupiáñez, Weaver, } \\
\text { Tipper, \& Madrid } \\
\text { (2001) }\end{array}$ & 2 & $20 \%$ & Color & $100,1,000$ & $\begin{array}{l}\text { different subjects for } \\
\text { each CTOA }\end{array}$ & 40 \\
\hline $\begin{array}{l}\text { Pratt, Kingstone, \& } \\
\text { Khoe (1997) }\end{array}$ & 1 & None & Identity $(\mathrm{X} /+)$ & $960 *$ & None & 16 \\
\hline
\end{tabular}

Note-CTOA, Cue-target onset asynchrony. *This experiment was included in the 1,000-msec CTOA analysis; we thank Jay Pratt for providing this data.

CTOA was analyzed separately because individual subjects contributed to all, some, or only one of the CTOAs. $\mathrm{T}-\mathrm{R}$ compatibility and cuing were the within-subjects factors and experiment was the between-subjects factor. Only experiments conducted within a single session and without distractors were included in the analysis. The between-experiments differences included discrimina- tion type (color or identity), the percentage of catch trials, stimuli eccentricity, stimuli duration, use of a central cue (so that attention returns to fixation), and eye monitoring.

The results of the repeated-measures analyses of variances (ANOVAs) are shown in Table 2. Figure 2 shows the mean RTs as a function of CTOA, target location, and

Table 2

Analyses of Variance for Each Cue-Target Onset Asynchrony (CTOA)

\begin{tabular}{|c|c|c|c|c|c|c|}
\hline CTOA & Factor & $d f_{\text {factor }}$ & $d f_{\text {error }}$ & $F$ ratio & $p$ value & Power \\
\hline \multirow[t]{7}{*}{100} & Experiment & 11 & 191 & 3.31 & $<.001$ & .99 \\
\hline & $\mathrm{T}-\mathrm{R}$ compatibility & 1 & 191 & 10.75 & .001 & .92 \\
\hline & Experiment $\times \mathrm{T}-\mathrm{R}$ compatibility & 11 & 191 & 0.68 & .756 & .37 \\
\hline & Cuing & 1 & 191 & 146.55 & $<.001$ & 1.00 \\
\hline & Experiment $\times$ cuing & 11 & 191 & 2.08 & .024 & .91 \\
\hline & $\mathrm{T}-\mathrm{R}$ compatibility $\times$ cuing & 1 & 191 & 0.17 & .680 & .07 \\
\hline & Experiment $\times \mathrm{T}-\mathrm{R}$ compatibility $\times$ cuing & 11 & 191 & 1.71 & .073 & .83 \\
\hline \multirow[t]{7}{*}{400} & Experiment & 5 & 101 & 5.10 & $<.001$ & .99 \\
\hline & $\mathrm{T}-\mathrm{R}$ compatibility & 1 & 101 & 1.95 & .166 & .27 \\
\hline & Experiment $\times \mathrm{T}-\mathrm{R}$ compatibility & 5 & 101 & 2.00 & .085 & .65 \\
\hline & Cuing & 1 & 101 & 21.70 & $<.001$ & 1.00 \\
\hline & Experiment $\times$ cuing & 5 & 101 & 1.81 & .118 & .59 \\
\hline & $\mathrm{T}-\mathrm{R}$ compatibility $\times$ cuing & 1 & 101 & 0.02 & .901 & .05 \\
\hline & Experiment $\times \mathrm{T}-\mathrm{R}$ compatibility $\times$ cuing & 5 & 101 & 0.98 & .435 & .33 \\
\hline \multirow[t]{7}{*}{700} & Experiment & 4 & 96 & 3.89 & .006 & .89 \\
\hline & $\mathrm{T}-\mathrm{R}$ compatibility & 1 & 96 & 11.93 & $<.001$ & .95 \\
\hline & Experiment $\times \mathrm{T}-\mathrm{R}$ compatibility & 4 & 96 & 2.66 & .037 & .72 \\
\hline & Cuing & 1 & 96 & 18.57 & $<.001$ & 1.00 \\
\hline & Experiment $\times$ cuing & 4 & 96 & 1.80 & .136 & .52 \\
\hline & $\mathrm{T}-\mathrm{R}$ compatibility $\times$ cuing & 1 & 96 & 0.73 & .397 & .13 \\
\hline & Experiment $\times \mathrm{T}-\mathrm{R}$ compatibility $\times$ cuing & 4 & 96 & 0.63 & .642 & .20 \\
\hline \multirow[t]{7}{*}{1,000} & Experiment & 8 & 150 & 4.34 & $<.001$ & 1.00 \\
\hline & $\mathrm{T}-\mathrm{R}$ compatibility & 1 & 150 & 21.03 & $<.001$ & 1.00 \\
\hline & Experiment $\times \mathrm{T}-\mathrm{R}$ compatibility & 8 & 150 & 1.15 & .331 & .52 \\
\hline & Cuing & 1 & 150 & 31.63 & $<.001$ & 1.00 \\
\hline & Experiment $\times$ cuing & 8 & 150 & 1.34 & .230 & .59 \\
\hline & $\mathrm{T}-\mathrm{R}$ compatibility $\times$ cuing & 1 & 150 & 10.20 & .002 & .91 \\
\hline & Experiment $\times \mathrm{T}-\mathrm{R}$ compatibility $\times$ cuing & 8 & 150 & 1.36 & .2199 & .60 \\
\hline
\end{tabular}

Note-T-R, target-response. 


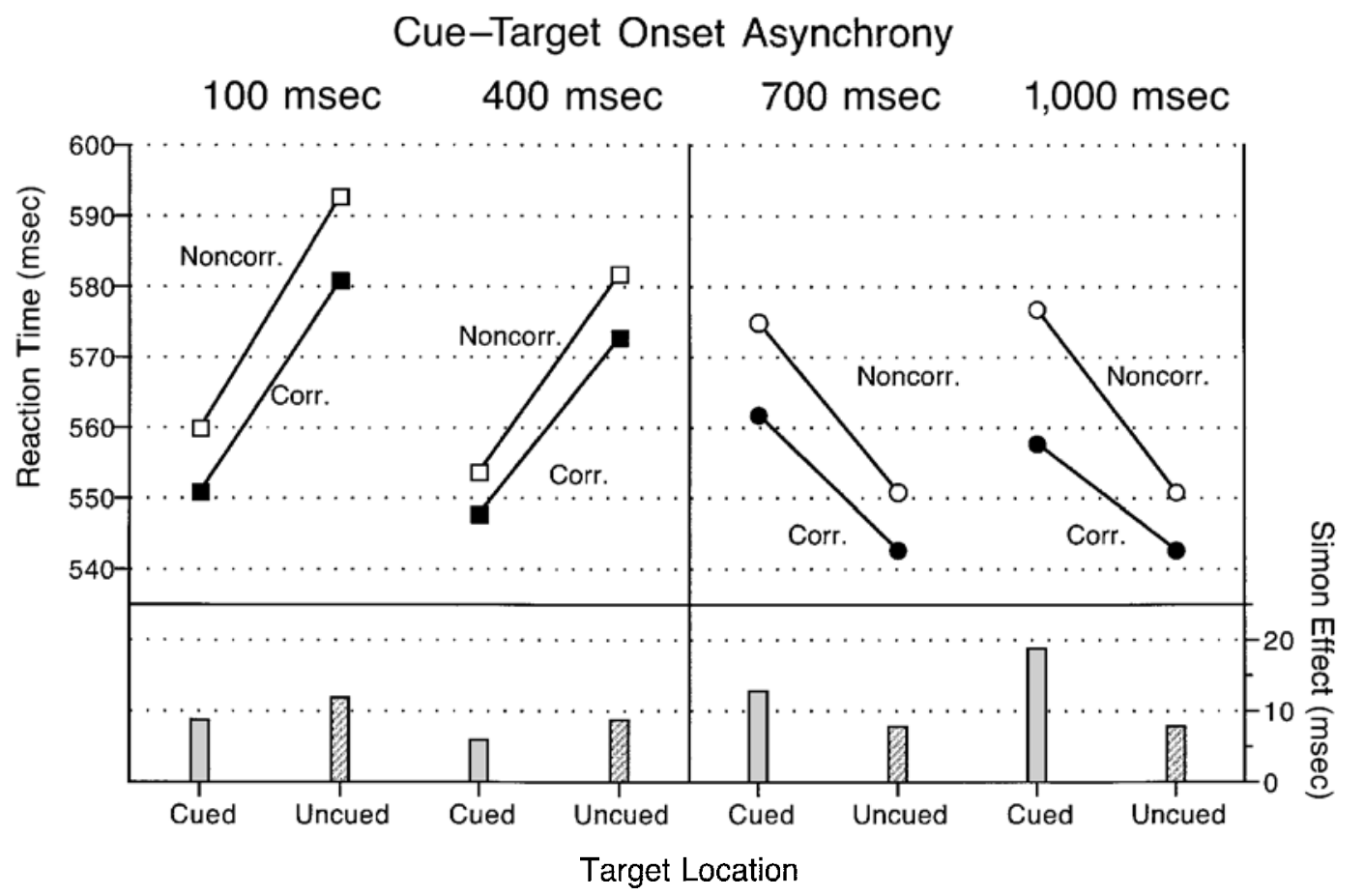

\begin{abstract}
Figure 2. Mean reaction time (in msec) as a function of cue condition, target-response (T-R) correspondence, and cue-target onset asynchrony (CTOA). The bars at the bottom of the figure show the size of the Simon effect (noncorrespondence RT minus correspondence RT) at the cued (stippled bars) and uncued (hatched bars) locations.
\end{abstract}

T-R compatibility. The magnitudes of the Simon effect ( $\mathrm{T}-\mathrm{R}$ noncorrespondence minus $\mathrm{T}-\mathrm{R}$ correspondence) as a function of CTOA and cuing are shown as bars on the bottom of Figure 2. We will focus on cuing, T-R compatibility, and the interaction between cuing and $\mathrm{T}-\mathrm{R}$ compatibility. The longest $(1,000 \mathrm{msec})$ and shortest (100 msec) CTOAs will be emphasized both because the largest number of subjects were tested in these conditions (over 150 compared with about 100 in the 400 - and 700-msec CTOA conditions, respectively) and because the facilitative and inhibitory effects of the cues are expected to be largest at these intervals.

\section{Short CTOAs}

At the 100-msec CTOA, there is significant cue facilitation $(32 \mathrm{msec})$, a significant Simon effect $(11 \mathrm{msec})$, and a nonsignificant interaction between the Simon effect and facilitation. One might wonder whether the difference between the Simon effect at the cued location $(9 \mathrm{msec})$ and at the uncued location $(12 \mathrm{msec})$ might be consistent with some reports that the Simon effect is reduced at an attended location (the attention-shift account of the Simon effect has been reviewed by Stoffer \& Umiltà, 1997). Is it possible that the interaction is real but not significant because of a lack of statistical power? To explore this possibility using the most unambiguous data, we excluded subjects who did not show facilitation on both T-R compatible and T-R incompatible trials. When this analysis was confined to subjects whose data unambiguously indicated that the cued location was attended, the Simon effect was $15 \mathrm{msec}$ at the cued location and $15 \mathrm{msec}$ at the uncued location, and the interaction between the Simon effect and cuing was nonsignificant $[F(1,115)=0.0418, p=.893$, power $=.052]$. Therefore, it is unlikely that the Simon effect is substantially altered by a prior shift of attention to the location of the target. This finding is problematic for the attention-shift hypothesis of the Simon effect, perhaps because a shift of attention is not sufficient for generating a spatial stimulus code (Ivanoff \& Peters, 2000).

Visual inspection of the data in Figure 2 reveals that the pattern of results in the 400-msec CTOA condition was very similar to that in the $100-$ msec CTOA condition. However, at this CTOA, the facilitation $(26.5 \mathrm{msec})$, but not the Simon effect ( $7.5 \mathrm{msec}$ ), was significant. Unfortunately, a nonsignificant effect is not especially meaningful. Subsequent analyses strongly argue against accepting the null hypothesis as a nil effect. ${ }^{3}$ Given that the primary purpose of this investigation was to examine the possibility of an interaction between the IOR and Simon effects, the weak Simon effect at a CTOA where facilitation is observed can remain a puzzle for future experimentation.

\section{Long CTOAs}

At the 1,000-msec CTOA, there was a significant IOR effect $(20.5 \mathrm{msec})$ and a significant Simon effect (13.5 $\mathrm{msec}$ ). In addition, there was a significant interaction be- 
tween these effects: The Simon effect was larger for targets at the cued than at the uncued location. When we analyzed each experiment separately at the $1,000-\mathrm{msec}$ CTOA, 7 out of 8 times the interaction was nonsignificant. When we excluded from the analysis the one experiment in which the interaction was significant (Lupiáñez et al., 1997, Experiment 3B), the interaction between the Simon and IOR effects remained significant $[F(1,133)=6.62, p=.011]$. This analysis shows that the interaction in our meta-analysis of the literature was not due to the influence of one particular experiment.

An inspection of the results, shown in Figure 2, reveals a pattern in the 700-msec CTOA condition that is very similar to that in the 1,000 -msec CTOA condition. In the 700-msec SOA condition, the inhibitory effect of the cue $(21.5 \mathrm{msec})$ and the Simon effect $(10.5 \mathrm{msec})$ were significant, but the interaction, although similar in magnitude to that in the 1,000-msec CTOA, was not. Because the data at these two longer CTOAs, at which an IOR effect was observed, are so similar, and because of the combined analyses (described in the next paragraph), it would be inappropriate to accept the lack of a significant interaction at the 700-msec CTOA as evidence of additivity.

The data from all the subjects who were tested at the 700 -msec SOA $(n=101)$ and the data of the subjects who were tested at 1,000 but not $700 \mathrm{msec}(n=76)$ were submitted to an ANOVA with cuing and T-R compatibility as the within-subjects factors and CTOA as the between-subjects factor. The IOR and the Simon effects were significant as was their interaction $[F(1,175)=$ $7.211, p<.01]$. The three-way interaction with CTOA was not significant $[F(1,175)=1.893, p=.171$, power $=$ $.262]$, suggesting that the interaction between the IOR effect and the Simon effect is not necessarily CTOA dependent. However, the absence of an interaction cannot be used to support the idea that the interaction does not change with CTOA. Likelihood ratios (LRs; Dixon, 1998, see note 3; Dixon \& O'Reilly, 1999) were used to compare a description of the data that assumes that the Simon effect is not different at the cued and uncued location versus a description that assumes that the Simon effect is different at the cued and uncued location. Because CTOA varied both between subjects and within subjects, we eliminated some of the intersubject variability by examining Simon effects (T-R noncorrespondence RTs minus T-R correspondence RTs) as a function of cue condition. At the 700-msec CTOA, the LR was 1.89 and at the $1,000-\mathrm{msec}$ CTOA, the LR was 22.65. In both situations, a description of the data that included the cue condition fared better than a description that did not, although the advantage was small at the 700-msec CTOA. A benefit of LRs is that they may be pooled across different experiments (Dixon, 1998). Thus, if we pooled the two CTOAs, the LR is 42.73 . Given the data, a description that holds that IOR increases the Simon effect is 42.73 times more likely than one that assumes that the Simon effect is unaltered by
IOR. Thus, the Simon and IOR effects do interact, but the effect size of the interaction is small, presumably because of an unusual amount of performance variability. For this reason, a large amount of power is required to detect it.

\section{Discussion}

The empirical contribution of our omnibus analysis is apparent in Figure 2: The Simon effect was larger at the cued location at the longer CTOAs, whereas facilitation (at the short CTOAs) had little impact on the Simon effect. The nonsignificant interaction between facilitation and the Simon effect replicates previous observations (Hommel, 1993b; Proctor, Lu, \& Van Zandt, 1992; Zimba $\&$ Brito, 1995) and suggests that a prior shift of attention to the target's location is not sufficient to modify the Simon effect. ${ }^{4}$ In contrast, the omnibus analyses clearly shows that the later "inhibitory" effect of the cue significantly increased the Simon effect. The presence of an interaction between the IOR and Simon effects is clearly confirmed here. This provides support for the proposal that the Simon and IOR effects share a common processing stage (e.g., response selection); however, it does not, by itself, suggest how IOR alters the Simon effect.

To understand the influence of IOR on the Simon effect, we have adopted the dual-process explanation of the Simon effect (e.g., De Jong et al., 1994; Hommel, 1993a, 1994; Kornblum \& Lee, 1995; Kornblum et al., 1999). According to this generally accepted construal of the Simon effect, responses in a Simon task are the product of an S-R code based on the task-irrelevant location of the target and an $\mathrm{S}-\mathrm{R}$ code based on the relevant identity of the target. The task-irrelevant spatial code is thought to facilitate the generation of a spatially corresponding response and impede the generation of a noncorresponding response. Figures $3 \mathrm{~A}$ and $3 \mathrm{~B}$ illustrate these hypothetical components of the Simon effect. One key assumption is that the magnitude of the Simon effect is proportional to the level of covert $\mathrm{S}-\mathrm{R}$ code activation from the target's task-irrelevant location at the time the correct response is selected. This activation process is assumed to increase from the time of target presentation until some maximum is reached and then to decay (De Jong et al., 1994; Hommel, 1994). In order to explore the impact of cuing on RT to corresponding and noncorresponding targets, we have assumed in Figure 3C that a response will be initiated when the net activation exceeds some criterion.

The IOR effect occurs in simple-RT (e.g., detection) tasks in which the Simon effect is virtually absent (e.g., Ivanoff \& Klein, 2001) and when the task does not call for a spatial response (Klein \& Dick, in press). Thus, before we consider how IOR might influence the Simon effect, it is necessary to propose mechanisms that would cause an RT delay for cued targets at relatively long CTOAs.

We will consider two broad theories of IOR that can explain its presence in simple-RT and Simon-type choice-RT 

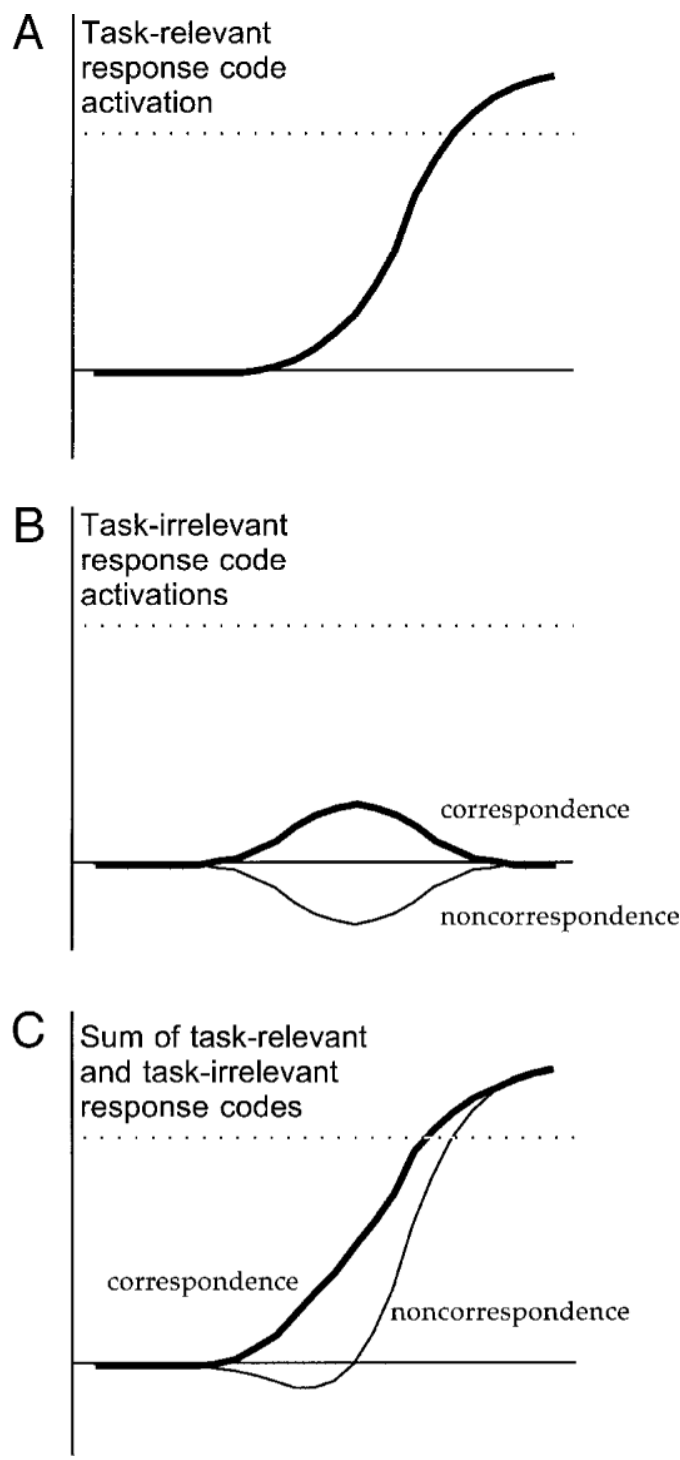

Figure 3. An illustration of the hypothetical components in a Simon task. The abscissa represents time, and the ordinate represents activation. (A) The activation of the task-relevant $S-R$ code increases until a threshold is reached and a response is initiated. (B) The task-irrelevant location of the target is thought to activate the response that spatially corresponds to the target's location. Two such activations are shown, one of which corresponds to the correct response (thick line) and one of which does not (thin line). This activation reaches a maximum and then decays. (C) The summation of (A) and (B).

tasks: the inhibition account ${ }^{5}$ (this is similar to the Fuentes et al., 1999, temporary $S-R$ disconnection hypothesis $^{6}$ and to Posner \& Cohen's, 1984, inhibited attention hypothesis) and the criterion-shift account (Klein \& Taylor, 1994). These distinct, but not mutually exclusive, mechanisms are conceptually illustrated along the top row of Figure 4. According to an inhibition account (Figure 4A), slower RTs to cued targets result from a delay, relative to uncued targets, in the activation of the task- relevant S-R code. Although such a delay could begin with the earliest levels of target processing (see, e.g., Handy, Jha, \& Mangun, 1999; McDonald, Ward, \& Kiehl, 1999; but see Klein, Schmidt, \& Müller, 1998, for evidence against this idea), for present purposes it only matters that the activation of the task-relevant response code is delayed. The function representing the accumulation of task-relevant information is shifted rightward so that to achieve the same level of accuracy, RTs must be slower. According to the criterion-shift account (Figure 4B), IOR reflects a reluctance to respond to targets at the cued location (Ivanoff \& Klein, 2001; Klein \& Taylor, 1994). Although the accumulation of information is unaffected at the cued location, an RT delay occurs because the criterion for initiating a response to targets in the cued region is raised. In the context of these two broadly defined accounts of IOR, in conjunction with the construal of the Simon effect illustrated in Figure 3, we will consider seven descriptions ${ }^{7}$ (shown in the far-left column in Figure 4) of the effect that IOR might have on the activation of the irrelevant $S-R$ code that underlies the Simon effect. This yields 14 predictions regarding the interaction between the IOR effect and the Simon effect.

\section{IOR Has No Effect on the Activation of the Task-Irrelevant S-R Code (Figure 4-1)}

The first view to consider is that IOR has no effect on the activation of the task-irrelevant spatial S-R code. In the context of both accounts of IOR (i.e., inhibition and criterion shift), this view of IOR predicts that the Simon effect will be smaller at the cued location. To slow the relevant S-R code (Figure 4-1A) or to increase the criterion for a response (Figure 4-1B) would allow the irrelevant $\mathrm{S}-\mathrm{R}$ code time to decay and, therefore, the Simon effect would be reduced at the cued location. The results of our omnibus analysis show that the Simon effect is larger, not smaller, at the cued location (Figure 2). Therefore, IOR must have some effect on the activation of the irrelevant $\mathrm{S}-\mathrm{R}$ code. Next we will consider six possible effects of IOR on the irrelevant $\mathrm{S}-\mathrm{R}$ code. ${ }^{8}$

\section{IOR Causes a Delay in the Activation of the Task-Irrelevant S-R Code (Figure 4-2)}

According to the inhibition account of IOR, the natural assumption would be that IOR delays the activation of all codes generated by stimuli presented in the cued region. Indeed, if IOR delayed the relevant and irrelevant $\mathrm{S}-\mathrm{R}$ codes by the same amount, the additive pattern that has been incorrectly accepted until now should be predicted (Figure 4-2A). The actual form of the interaction between the Simon and IOR effects (Figure 2) allows us to reject this account. In contrast, when combined with the criterion-shift explanation of IOR, the view that IOR delays the activation of the irrelevant $\mathrm{S}-\mathrm{R}$ code predicts the interaction we have observed, with a larger Simon effect at the cued location (Figure 4-2B). It is interesting to consider the additive relation between cuing and the Simon effect when the cue-target interval is short enough 
Figure 4. Illustrated are 14 predictions resulting from the combination of the inhibition (A) and criterion-shift (B) theories of IOR with seven construals of how IOR might affect the task-irrelevant spatial $S-R$ code: (1) IOR does not affect the task-irrelevant S-R code, (2) IOR delays the onset of the task-irrelevant $S-R$ code, $(3)$ IOR accelerates the onset of the task-irrelevant $S-R$ code, (4) IOR increases the activation of the task-irrelevant $S-R$ code, (5) IOR decreases the activation of the task-irrelevant $S-R$ code, (6) IOR delays the suppression of the task-irrelevant $S-R$ code's activation, or (7) IOR accelerates the suppression of the taskirrelevant $S-R$ code's activation. The cells of the predictions that do not match the actual results are boxed. Target-response correspondence is represented by the thin lines and target-response noncorrespondence by the thick lines. See text for details.

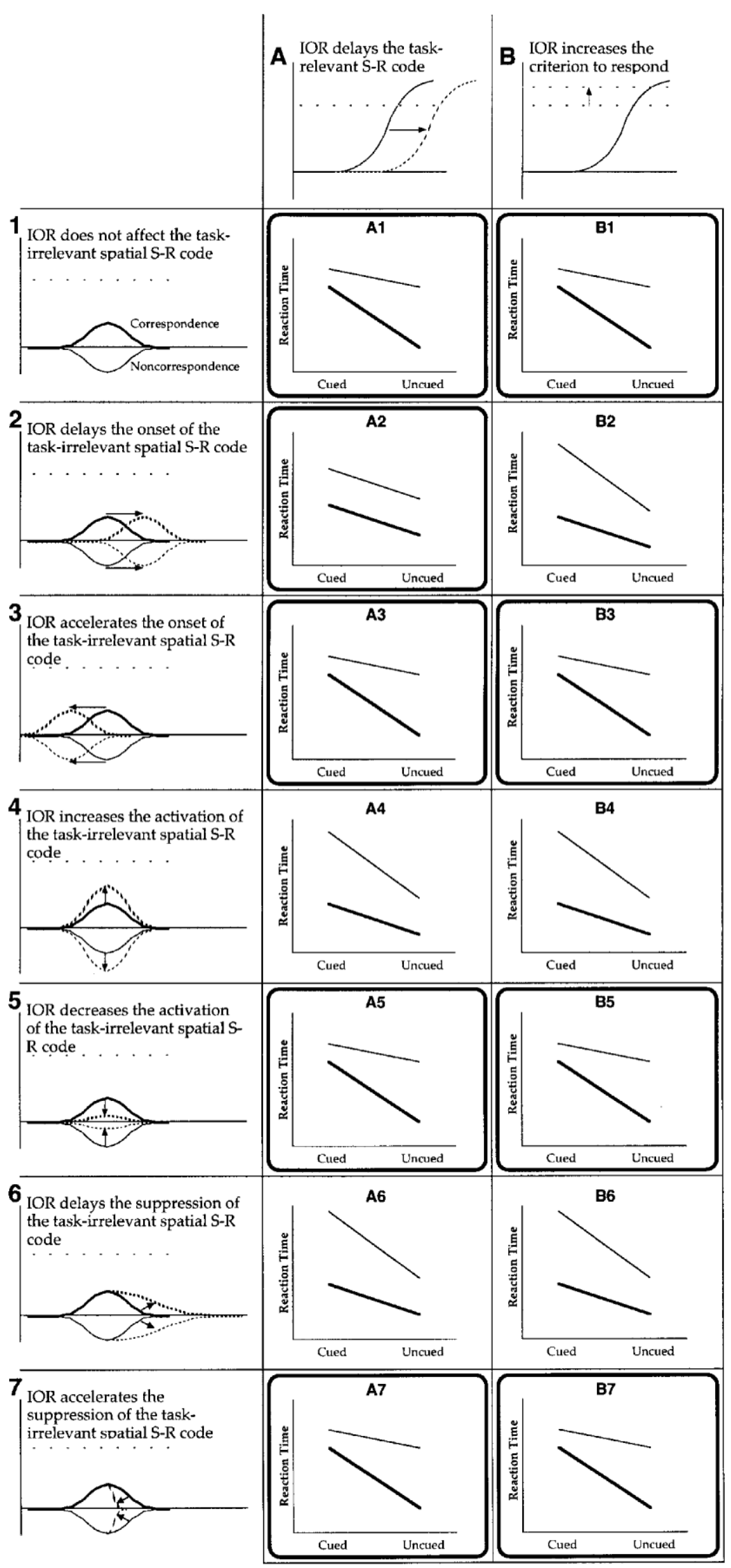


to produce facilitation. This is precisely what would be expected if the facilitation associated with exogenous orienting accelerated the functions for both the relevant and irrelevant codes equally.

\section{IOR Accelerates the Onset of the Activation of the Task-Irrelevant S-R Code (Figure 4-3)}

If IOR temporally shifts the onset of the irrelevant $S-R$ code to an earlier point in time, the Simon effect will be reduced at the cued location. This prediction holds whether or not one adopts the view that IOR delays the activation of the relevant S-R code (Figure 4-3A) or increases the criterion to respond at the cued location (Figure 4-3B). The results, as shown in Figure 2, clearly do not endorse either view.

\section{IOR Increases the Activation of the Task-Irrelevant S-R Code (Figure 4-4)}

Regardless of the general account adopted for IOR (i.e., inhibition or criterion shift), the increase in the activation of the irrelevant $S-R$ code results in an increase in the Simon effect and hence an interaction of the form seen in Figure 2 (Figures 4-4A,B).

\section{IOR Decreases the Activation of the}

\section{Task-Irrelevant S-R Code (Figure 4-5).}

According to both theories of the effect that IOR has on the task-relevant $\mathrm{S}-\mathrm{R}$ code, the reduction in the activation of the irrelevant $S-R$ code results in a smaller Simon effect. The actual results do not support the predictions of this hypothesis.

\section{IOR Causes a Delay in the Decay of the Activation of the Task-Irrelevant S-R Code (Figure 4-6)}

In Figure 4-4, we have illustrated an increase in the irrelevant spatial $\mathrm{S}-\mathrm{R}$ code without a change in its time course. However, an increase at longer times might be produced if IOR slowed or temporarily delayed the decay of the task-irrelevant S-R code (Figure 4-6). For both theories of IOR, this will also result in an increase in the Simon effect at the cued location. The results do not falsify this hypothesis.

\section{IOR Accelerates the Delay of the Decay of the Activation of the Task-Irrelevant S-R Code (Figure 4-7)}

The acceleration of the rate of irrelevant $S-R$ code decay, like the decrease in the activation of the irrelevant S-R code (Figure 4-5), should reduce the Simon effect at the cued location. The results do not support this idea.

\section{CONCLUSION}

Our reanalysis of the relationship between the Simon and IOR effects unambiguously demonstrates an interaction. At long intervals, when the IOR effect was observed, the Simon effect was twice as large at the cued location than at the uncued location. This observation is particularly compelling because several manipulations that delay reaction time have been shown to decrease the Simon effect (Hommel, 1993a, 1994; for a similar demonstration, see De Jong et al., 1994). However, IOR, which delays RT, increases the Simon effect. If the slowing of RTs, due to IOR, partly reduces the Simon effect whereas IOR also increases it, the interaction will be very noisy and thus difficult to detect statistically. Thus, whether or not one accepts the interpretations of the Simon and IOR effects that we have considered, the form of the interaction between cuing and T-R compatibility at long CTOAs stands as a bonafide and robust empirical pattern. ${ }^{9}$

By using the most generally accepted interpretation of the Simon effect and two broadly drawn interpretations of IOR's effects, we were able to consider 14 hypotheses concerning the interaction between these effects. In this context, which is illustrated in Figure 4, we were able to reject 9 of the 14 hypotheses. Broadbent (1958), arguing against the hypothetico-deductive method, noted that scientific progress would be much more rapid if we conducted experiments that split the potential theories into two, roughly equal, groups, and provided support for one group and against the other. Studies that have combined exogenous cuing with T-R compatibility, such as those included in our analysis, provide-serendipitously perhaps - the kind of evidence Broadbent championed. Regardless of the interpretation one adopts for the RT delay associated with IOR, we can reject the proposals that: (1) IOR has no effect on the activation of the taskirrelevant S-R code (Figure 4-1), (2) IOR accelerates the decay of the task-irrelevant S-R code (Figure 4-7), (3) IOR accelerates the onset of the task-irrelevant $\mathrm{S}-\mathrm{R}$ code's activation (Figure 4-3), and (4) IOR reduces the task-irrelevant $\mathrm{S}-\mathrm{R}$ code activation (Figure 4-5). If IOR is assumed to delay the activation of the relevant $\mathrm{S}-\mathrm{R}$ code, we can also reject the proposal that IOR delays the irrelevant activation by a similar (or smaller) amount (Figure 4-2A). The remaining interpretations (IOR increases [Figure 4-4] or delays the suppression [Figure 4-6] of the irrelevant $\mathrm{S}-\mathrm{R}$ code; or, if the main mechanism of IOR reflects a response bias, it may also delay this activation [Figure 4B] ) are all consistent with an increase in the Simon effect at the cued location. We believe that further progress in choosing among these alternatives will depend on methods such as the generation of speed-accuracy tradeoff functions (Wickelgren, 1977) and the recording of event-related brain activity (e.g., De Jong et al., 1994; Eimer, 1998; Mouret \& Hasbroucq, 2000), which can provide more direct evidence of the time course of $\mathrm{S}-\mathrm{R}$ code activations.

\section{REFERENCES}

Abrams, R. A., \& Dob KIn, R. S. (1994). Inhibition of return: Effects of attentional cuing on eye movement latencies. Journal of Experimental Psychology: Human Perception \& Performance, 20, 467-477. Broadbent, D. E. (1958). Perception and communication. Oxford: Pergamon.

De Jong, R., Liang. C.-C., \& Lauber, E. (1994). Conditional and un- 
conditional automaticity: A dual-process model of effects of spatial stimulus-response correspondence. Journal of Experimental Psychology: Human Perception \& Performance, 20, 731-750.

Dixon, P. (1998). Why scientists value $p$ values. Psychonomic Bulletin \& Review, 5, 390-396.

Dixon, P., \& O’Reilly, T. (1999). Scientific versus statistical inference. Canadian Journal of Experimental Psychology, 53, 133-149.

Eimer, M. (1998). The lateralized readiness potential as an on-line measure of central response activation processes. Behavior Research Methods, Instruments, \& Computers, 30, 146-156.

Fuentes, L. J., Vivas, A. B., \& Humphreys, G. W. (1999). Inhibitory tagging of stimulus properties in inhibition of return: Effects on semantic priming and flanker interference. Quarterly Journal of Experimental Psychology, 52A, 149-164.

Handy, T. C., Jha, A. P., \& MAngun, G. R. (1999). Promoting novelty in vision: Inhibition of return modulates perceptual-level processing. Psychological Science, 10, 157-161.

Hommel, B. (1993a). The relationship between stimulus processing and response selection in the Simon task: Evidence for a temporal overlap. Psychological Research, 55, 280-290.

Hommel, B. (1993b). The role of attention for the Simon effect. Psychological Research, 55, 208-222.

Hommel, B. (1994). Spontaneous decay of response-code activation. Psychological Research, 56, 261-268.

IvANOFF, J., \& KLEIN, R. M. (2001). The presence of a nonresponding effector increases inhibition of return. Psychonomic Bulletin \& Review, 8, 307-314.

Ivanoff, J., \& Peters, M. (2000). A shift of attention may be necessary, but it is not sufficient, for the generation of the Simon effect. Psychological Research, 64, 117-135.

KLEIN, R. M. (1988). Inhibitory tagging system facilitates visual search. Nature, 334, 430-431.

KLEIN, R. M. (2000). Inhibition of return. Trends in Cognitive Sciences, 4, 138-146.

KLEIN, R. M., \& Dick, B. (in press). RSVP exploration of the temporal dynamics of reflexive attention shifts. Psychological Science.

Klein, R. M., Schmidt, W. C., \& Müller, H. J. (1998). Disinhibition of return: Unnecessary and unlikely. Perception \& Psychophysics, 60, 862-872.

KLeIN, R. M., \& TAYLOR, T. L. (1994). Categories of cognitive inhibition, with reference to attention. In D. Dagenbach \& T. H. Carr (Eds.), Inhibitory processes in attention, memory, and language (pp. 113-150). San Diego: Academic Press.

Kornblum, S., Hasbroucq, T., \& Osman, A. (1990). Dimensional overlap: Cognitive basis for stimulus-response compatibility: A model and taxonomy. Psychological Review, 97, 253-270.

Kornblum, S., \& LEE, J.-W. (1995). Stimulus-response compatibility with relevant and irrelevant stimulus dimensions that do and do not overlap with the response. Journal of Experimental Psychology: Human Perception \& Performance, 21, 855-875.

Kornblum, S., Stevens, G. T., Whipple, A., \& Requin, J. (1999). The effects of irrelevant stimuli: 1 . The time course of stimulus-stimulus and stimulus-response consistency effects with Stroop-like stimuli, Simon-like tasks, and their factorial combinations. Journal of Experimental Psychology: Human Perception \& Performance, 25, 688714.

Lu, C.-H., \& Proctor, R. W. (1995). The influence of irrelevant location information on performance: A review of the Simon and spatial Stroop effects. Psychonomic Bulletin \& Review, 2, 174-207.

Lupiáñez, J., Milán, E. G., Tornay, F. J., Madrid, E., \& Tudela, P. (1997). Does IOR occur in discrimination tasks? Yes, it does, but later. Perception \& Psychophysics, 59, 1241-1254.

Lupiáñez, J., \& Milliken, B. (1999). Inhibition of return and the attentional set for integrating versus differentiating information. Journal of General Psychology, 126, 392-418.

Lupiáñez, J., Milliken, B., Solano, C., Weaver, B., \& Tipper, S. P. (2001). On the strategic modulation of the time course of facilitation and inhibition of return. Quarterly Journal of Experimental Psychology, 54A, 753-773.

LuPIÁÑez, J., \& Solano, C. (1998). Inhibition of return in a color discrimination task: No interaction with the Simon effect. Cognitiva, 9 , 195-205.
Lupiáñez, J., Tudela, P., \& Rueda, C. (1999). Control inhibitorio en la orientacion atencional: Un revision sobre la inhibicion de retorno [Inhibitory control in attentional orientation: A review about the inhibition of return]. Cognitiva, 11, 23-44. [Abstract in English]

Lupiáñez, J., WeaVer, B., TipPer, S. P., \& Madrid, E. (2001). The effects of practice on cuing effects in detection and discrimination tasks. Psicológica, 22, 1-23.

McDonald, J. J., Ward, L. M., \& KIEHL, K. A. (1999). An event-related brain potential study of inhibition of return. Perception \& Psychophysics, 61, 1411-1423.

Mouret, I., \& HasbroucQ, T. (2000). The chronometry of single neuron activity: Testing discrete versus continuous models of information processing. Journal of Experimental Psychology: Human Perception \& Performance, 26, 1622-1638.

PosNer, M. [I.] (1980). Orienting of attention. Quarterly Journal of Experimental Psychology, 32, 3-25.

Posner, M. I., \& COHEN, Y. (1984). Components of visual orienting. In H. Bouma \& D. G. Bouwhuis (Eds.), Attention and performance $X$ (pp. 531-556). Hillsdale, NJ: Erlbaum.

Posner, M. I., Rafal, R. D., Choate, L. S., \& Vaughan, J. (1985). Inhibition of return: Neural basis and function. Cognitive Neuropsychology, 2, 211-228.

Pratt, J., Kingstone, A., \& Khoe, W. (1997). Inhibition of return in location- and identity-based choice decision tasks. Perception \& Psychophysics, 59, 964-971.

Proctor, R. W., Lu, C.-H., \& VAn ZANDT, T. (1992). Enhancement of the Simon effect by response precuing. Acta Psychologica, 74, 53-74.

Sheliga, B. M., Craighero, L., Riggio, L., \& Rizzolatti, G. (1997). Effects of spatial attention on directional manual and ocular responses. Experimental Brain Research, 114, 339-351.

STERNBERG, S. (1969). The discovery of processing stages: Extension of Donders' method. In W. G. Koster (Ed.), Attention and performance II: Proceedings of the Donders centenary symposium on reaction time. Amsterdam: North-Holland.

Stoffer, T. H., \& UMiLtà, C. (1997). Spatial stimulus coding and the focus of attention in S-R compatibility and the Simon effect. In B. Hommel, \& W. Prinz (Eds.), Theoretical issues in $S-R$ compatibility (pp. 181-208). Amsterdam: North-Holland.

Stoffer, T. H., \& YAKIN, A. R. (1994). The functional role of attention for spatial coding in the Simon effect. Psychological Research, 56, 151-162.

TAYLOR, T. L., \& KLEIN, R. M. (1998). On the causes and effects of inhibition of return. Psychonomic Bulletin \& Review, 5, 625-643.

TAYLOR, T. L., \& KLEIN, R. M. (2000). Visual and motor effects in inhibition of return. Journal of Experimental Psychology: Human Perception \& Performance, 26, 1639-1656.

WiCKELGREN, W. (1977). Speed-accuracy tradeoff and information processing dynamics. Acta Psychologica, 41, 67-85.

Zimba, L. D., \& Brito, C. F. (1995). Attention precuing and Simon effects: A test of the attention-coding account of the Simon effect. Psychological Research, 58, 102-118.

\section{NOTES}

1. Perhaps because the name of the effect includes its explanation, scholars have used IOR interchangeably to refer to an effect (cued RTs > uncued RTs, at relatively long CTOAs) and the mechanism that gives rise to the effect. We will use the term IOR effect when we refer to the pattern of results attributable to the underlying mechanism that we call IOR. We will use these terms in this manner without necessarily endorsing the original proposal.

2 . In the simple $2 \times 2$ analysis we used in this context, an interaction is present if the Simon effect for uncued trials is statistically different from the Simon effect for cued trials. It should be noted, however, that this way of expressing the interaction (i.e., that IOR is altering the Simon effect) is equivalent to determining whether IOR for T-R corresponding trials is statistically different from IOR for T-R noncorresponding trials.

3. Likelihood ratios (LRs; see Dixon, 1998; Dixon \& O'Reilly, 1999) were calculated in order to assess whether a description of the data that includes the Simon effect and facilitation fared better than a description that includes only facilitation. Although $p$ values are properly interpreted as the probability of the data given that the null hypothesis is 
true, the LR reflects the ratio of the likelihoods of the data given one description of the true state of affairs over another. That the Simon effect was not significant with the 400 -msec CTOA is puzzling, but this null effect should not necessarily be taken to mean that it is not present. We contrasted two different descriptions of the data: one that holds that only facilitation is present with the 400-msec CTOA versus one that includes both main effects of cue and of T-R compatibility. The likelihood ratio was 27.6. Dixon has argued that an LR of 10 or greater provides clear evidence in favor of one description over the other. Nonetheless, smaller LR values $(1<\mathrm{LR}<10)$ do provide some evidence in favor of one description over the other, but the evidence is not as strong. Thus, an LR of 27.61 means that the description of the data that includes the Simon effect fares better than one that does not include the Simon effect. Hence, although the Simon effect was not significant in the ANOVA, it would be imprudent to conclude that it was absent. Leading to the same conclusion, a more conventional analysis (cuing, T-R compatibility, CTOA, and experiment were factors) including the data from 107 subjects who were tested with the 100-msec and 400-msec CTOAs, where facilitation was observed, revealed a significant effect of T-R compatibility $[F(1,101)=8.351, p<.005$, power $=.831]$ and yet no interaction with CTOA $[F(1,101)=0.669, p=.416$, power $=.123]$. Whereas this nonsignificant interaction does not mean that the Simon effect is unaffected by CTOA, it cannot be interpreted to mean that it is.

4. This observation is important because it suggests that the Simon effect is unaffected by the destination or direction of an exogenous shift of attention. This finding appears to conflict with the conclusion drawn by several investigators (e.g., Sheliga, Craighero, Riggio, \& Rizzolatti, 1997; Stoffer \& Yakin, 1994) who found smaller Simon effects at a peripherally cued location than in their neutral conditions (see Hommel, 1993b, for a similar finding). The 9-msec Simon effect for valid cues with the 100-msec CTOA in this review is very close to the Simon effects for "valid" cues with CTOAs close to $100 \mathrm{msec}$ found by Stoffer and Yakin $(8 \mathrm{msec})$ and by Hommel (1993b; Experiment $5 ; 11 \mathrm{msec})$. Although Stoffer and Yakin used "traditional" peripheral cues, Hommel's (1993b) "valid" cue was the onset of the degraded target that was gradually presented during $196 \mathrm{msec}$, at which time the full stimulus was displayed. Stoffer and Yakin's neutral condition was a cue presented at center and Hommel's neutral condition (immediate condition) was a regular Simon task where the target was presented all at once. Stoffer and Yakin found a 32-msec Simon effect, and Hommel (1993b) found a 31-msec Simon effect in their respective neutral conditions. Note, however, that the Simon effect for uncued trials amounted to $12 \mathrm{msec}$ in our reanalysis. Uncued trials are like cued trials in that a shift of attention occurs before target onset. Because attention is not launched before the target's presentation with the neutral conditions employed by Stoffer and Yakin and Hommel (1993b), these neutral Simon effects do not constitute a fair comparison with the Simon effects for cued trials (Zimba \& Brito, 1995). Perhaps it is not that the Simon effect is reduced at an attended or cued location, but rather that the Simon effect is reduced by any shift of attention that precedes the target.

5 . We have opted to depict inhibition as a delay in the onset of the task-relevant $\mathrm{S}-\mathrm{R}$ code activation. Rather than just delay the onset of the relevant S-R code's activation, IOR might act to decelerate its acti- vation. Regardless of the precise nature of IOR's effect on the relevant $\mathrm{S}-\mathrm{R}$ code, the result on the Simon effect is the same (i.e., all other things being equal, the Simon effect would decrease).

6. Fuentes et al. (1999) found that the effect of an irrelevant flanker effect was reversed when the flanker was presented at the cued location. Although, on the basis of a comparison with a neutral flanker condition, Fuentes et al. concluded that IOR caused a temporary disconnection of stimuli from their associated responses, one might favor the equally viable reversal account for their pattern of results (i.e., IOR reversed the $\mathrm{S}-\mathrm{R}$ valence of the flanker). To discriminate between these accounts of the influence of IOR on the codes activated by irrelevant flankers will require further study.

7. Asking why IOR affects the irrelevant S-R code may be akin to asking why IOR occurs in the first place. Since Posner and Cohen (1984; see also Klein, 1988), it has been thought that IOR promotes visual search to new locations. Klein (1988) argued that inhibitory tags, which result from attention's leaving a recently visited location, prevent attention from returning to old locations. Perhaps these inhibitory tags are some kind of distortion in the mental representation of visuomotor space. If these tags are some kind of distortion, any of the scenarios in the far-left column of Figure 4 might suffice as a spatial tag. Although this is purely speculative, it does not matter in the approach that we have adopted here. The goal of this analysis was to determine the possible explanations that can account for the interaction between IOR and the Simon effect.

8. These six scenarios (far-left column of Figure 4) were selected because they seemed to be the most parsimonious ways that IOR might change the task-irrelevant spatial S-R code. This list is not exhaustive, however. One might imagine scenarios in which IOR increases or decreases the activation rate of the irrelevant spatial $\mathrm{S}-\mathrm{R}$ code. If this were the case, the apex of the irrelevant spatial S-R code would occur earlier or later than it would otherwise. Ultimately, an increase in the rate of the irrelevant spatial S-R code's activation would result in a reduction of the Simon effect, and a decrease in the rate of activation would result in an enhanced Simon effect. Thus, we have omitted the idea that IOR changes in the rate of the irrelevant spatial code's activation from Figure 4 because its increase or decrease would have an effect similar to the acceleration (Figure 4-3) or delay (Figure 4-2) of the activation of the irrelevant spatial code, respectively.

9. All of the experiments included in our review used two target locations, two cue locations, and two responses, all of which could be spatially coded as left or right. The reviewed studies were thus characterized by a high degree of dimensional overlap (i.e., conceptual, perceptual, or physical similarity between stimulus and response sets; Kornblum, Hasbroucq, \& Osman, 1990; Kornblum \& Lee, 1995). Whether the interaction between the Simon effect and IOR would be obtained under conditions with less dimensional overlap is a question for further research.

(Manuscript received February 29, 2000; revision accepted for publication June 14, 2001.) 